\title{
z-Knotted Triangulations of Surfaces
}

\author{
Mark Pankov ${ }^{1} \cdot$ Adam Tyc $^{2}$
}

Received: 23 May 2019 / Revised: 29 December 2019 / Accepted: 21 January 2020 /

Published online: 11 February 2020

(c) The Author(s) 2020

\begin{abstract}
A zigzag in a map (a 2-cell embedding of a connected graph in a connected closed 2dimensional surface) is a cyclic sequence of edges satisfying the following conditions: (1) any two consecutive edges lie on the same face and have a common vertex, (2) for any three consecutive edges the first and the third edges are disjoint and the face containing the first and the second edges is distinct from the face which contains the second and the third one. A map is $z$-knotted if it contains a single zigzag. Such maps are closely related to the Gauss code problem and have nice homological properties. We show that every triangulation of a connected closed 2-dimensional surface admits a $z$-knotted shredding.
\end{abstract}

Keywords Embeded graph · Triangulation · Zigzag

\section{Introduction}

Consider two adjacent edges $e_{1}$ and $e_{2}$ in a regular polyhedron. These edges have a common vertex $v$ and lie on the same face. We take the second face containing $e_{2}$. In this face, there is a unique edge $e_{3}$ intersecting $e_{2}$ in a vertex different from $v$. Similarly, we get an edge $e_{4}$ from the edges $e_{2}$ and $e_{3}$. Step by step, we create a sequence $e_{1}, e_{2}, e_{3}, \ldots$ whose elements will repeat after some time. Since we are dealing with a regular polyhedron, this is a skew polygon without self-intersections. Coxeter called such polygons Petrie polygons [1, Sect. 2.6]. They play an important

Editor in Charge: János Pach

Mark Pankov

pankov@matman.uwm.edu.pl

Adam Tyc

atyc@impan.pl

1 Faculty of Mathematics and Computer Science, University of Warmia and Mazury, Słoneczna 54, 10-710 Olsztyn, Poland

2 Institute of Mathematics, Polish Academy of Sciences, Śniadeckich 8, 00-656 Warsaw, Poland 


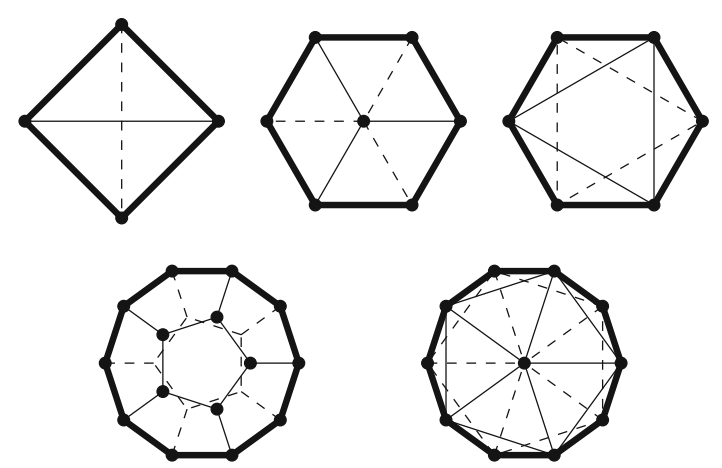

Fig. 1 Zigzags on the Platonic solids

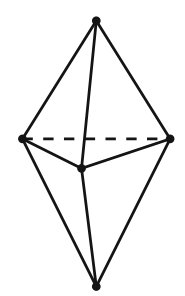

Fig. 2 3-gonal bipyramid

role in his famous book. See Fig. 1 for the Petrie polygons on the five Platonic solids (the bold line), observe that the dual solids have the same Petrie polygons.

In non-regular polyhedra, cyclic sequences with the property described above can have self-intersections. For example, a 3-gonal bipyramid (Fig. 2) has only one such sequence (up to reversing).

Analogues of Petrie polygons for maps, i.e., 2-cell embeddings of graphs in closed 2-dimensional surfaces, are known as zigzags [3,5] or closed left-right paths [4,9]. General results on zigzags in plane graphs can be found in [4, Chap. 17]. A large portion of material given in [3] concerns zigzags in three-regular plane graphs related to mathematical chemistry, in particular, well-known fullerenes. In both these books, the case when there is a single zigzag is distinguished. Following [3], we call maps satisfying this condition $z$-knotted.

A Gauss code is a word, where each symbol occurs precisely twice [4, Sect. 17.4]. There is a problem related to realizing Gauss codes as closed curves with simple selfintersections in closed 2-dimensional surfaces. It is not difficult to see that a map is $z$-knotted if and only if there is a zigzag passing trough each edge twice. The latter is equivalent to the fact that the corresponding medial graph (the graph whose vertex set is formed by all edges of the map, and two edges are adjacent if they have a common vertex and belong to the same face, see the bold graph in Fig. 3) is a realization of a certain Gauss code. We refer to [4, Sect. 17.7] for the case of plane graphs and to [2,6] for the general case.

The concept of $z$-knottedness can be described in terms of $\mathbb{Z}_{2}$-homologies. First of all, we observe that every zigzag is also a zigzag in the dual map and conversely. 


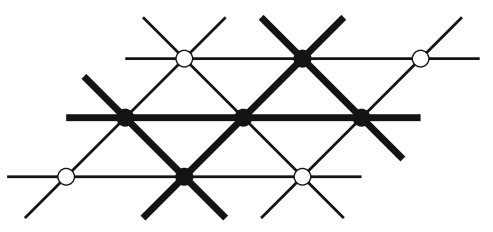

Fig. 3 Medial graph

If our map is not $z$-knotted, then there is a zigzag passing through some edges once. These edges form a bicycle, i.e., a cycle which is also a cycle in the dual map. By [4, Thm. 17.3.5], a plane graph is $z$-knotted if and only if it does not contain non-trivial bicycles. This is equivalent to the fact that the corank of the Laplacian over $\mathbb{Z}_{2}$ is equal to 1 and well-known Kirchhoff's theorem implies that a plane graph is $z$-knotted if and only if the number of spanning trees in this graph is odd [4, Sects. 14.15 and 17.8]. In the general case, we consider the vector space over $\mathbb{Z}_{2}$ formed by all bicycles with the symmetric difference as an additive operation. For a $z$-knotted map this vector space does not contain boundaries and coboundaries and its dimension is equal to the corresponding Betti number, i.e., there is a natural one-to-one correspondence between bicycles and 1-dimensional $\mathbb{Z}_{2}$-homologies [2, Sect. 4].

In this paper, we investigate zigzags in triangulations of closed 2-dimensional surfaces. It is well known that an $n$-gonal bipyramid is a $z$-knotted triangulation of the sphere $\mathbb{S}^{2}$ if $n$ is odd. Two examples of $z$-knotted fullerenes can be found in [3, Sect. 2.3] (the duals are $z$-knotted triangulations of $\mathbb{S}^{2}$ ). A large class of plane $z$-knotted graphs (in particular, $z$-knotted fullerenes) was obtained using computer [3].

Our main result (Theorem 3.1) states that every triangulation of any closed 2dimensional surface admits a $z$-knotted shredding.

This statement will be proved in two steps. First, we introduce the $z$-monodromy $M_{F}$ which acts on the set of oriented edges of a face $F$ in a triangulation. For two consecutive oriented edges $e_{0}$ and $e$ in $F$ we consider the unique zigzag containing the sequence $e_{0}, e$ and define $M_{F}(e)$ as the first (oriented) edge of $F$ which occurs in this zigzag after $e$. There are precisely seven possibilities for the $z$-monodromies and only four possibilities can be realized in $z$-knotted triangulations (Theorem 4.4). Examples show that each of these seven possibilities happens in a certain triangulation; moreover, the four possibilities corresponding to the $z$-knotted case are realized in $z$ knotted triangulations (Section 5). If the $z$-monodromies of all faces in a triangulation are of $z$-knotted type, then this triangulation is $z$-knotted (Theorem 4.7). The second step is a gluing lemma concerning zigzags in the connected sum of triangulations (Section 6). Using this lemma, we replace every face whose $z$-monodromy is not of $z$-knotted type by some number of faces with the $z$-monodromies of $z$-knotted types and get a $z$-knotted shredding.

Other application of the gluing lemma is a description of all cases when the connected sum of $z$-knotted triangulations is $z$-knotted (Theorem 8.1). 


\section{Zigzags in Triangulations}

We describe some elementary properties of zigzags in triangulations (which also hold for other maps).

Let $M$ be a connected closed 2-dimensional surface (not necessarily orientable) and let $\Gamma$ be a triangulation of $M$, i.e., a 2-cell embedding of a connected simple finite graph in $M$ whose faces are triangles [7, Sect. 3.1]. The following properties are immediate consequences of the definition: (1) every edge is contained in precisely two distinct faces, (2) the intersection of every two distinct faces is either an edge or a vertex, or it is empty.

Two distinct edges are called adjacent if there is a face containing them. Since every face is a triangle, any two adjacent edges have a common vertex. Two faces are said to be adjacent if their intersection is an edge.

A zigzag in $\Gamma$ is a sequence of edges $\left\{e_{i}\right\}_{i \in \mathbb{N}}$ satisfying the following conditions for every $i \in \mathbb{N}$ :

(Z1) $e_{i}, e_{i+1}$ are adjacent,

(Z2) the faces containing $e_{i}, e_{i+1}$ and $e_{i+1}, e_{i+2}$ are distinct and the edges $e_{i}$ and $e_{i+2}$ are disjoint.

Since $\Gamma$ is finite, for every zigzag $Z=\left\{e_{i}\right\}_{i \in \mathbb{N}}$ there is a natural number $n>0$ such that $e_{i+n}=e_{i}$ for every $i \in \mathbb{N}$. The smallest number $n$ satisfying this condition is called the length of $Z$. So, our zigzag is a cyclic sequence $e_{1}, \ldots, e_{n}$, where $n$ is the length of $Z$. We say that $Z$ is edge-simple if all edges in this cyclic sequence are mutually distinct. Note that $Z$ can be presented as a cyclic sequence of vertices $v_{1}, \ldots, v_{n}$, where $v_{i}$ and $v_{i+1}$ are the vertices belonging to $e_{i}$ for $i<n$ and the edge $e_{n}$ contains $v_{n}$ and $v_{1}$. The zigzag $Z$ is called simple if these vertices are mutually distinct. All zigzags of the Platonic solids are simple (see Fig. 1). It is clear that a simple zigzag is edge-simple, but an edge-simple zigzag is not necessarily simple.

Observe that every zigzag $\left\{e_{i}\right\}_{i \in \mathbb{N}}$ is completely determined by any pair of consecutive edges $e_{i}, e_{i+1}$. If $X=\left\{e_{1}, \ldots, e_{n}\right\}$ is a sequence of edges, then $X^{-1}$ denotes the reversed sequence $e_{n}, \ldots, e_{1}$. If $Z$ is a zigzag, then the same holds for $Z^{-1}$. If $Z$ contains a sequence $e, e^{\prime}$, then the sequence $e^{\prime}, e$ is contained in the reversed zigzag $Z^{-1}$. Sequences of type $e, e^{\prime}, \ldots, e^{\prime}, e$ are not contained in zigzags, i.e., a zigzag cannot be reversed to itself. Indeed, if such a sequence were contained in a zigzag, then this zigzag would be one of the following sequences;

$$
e, e^{\prime}, e_{1}, e_{2}, \ldots, e_{m}, e_{m}, \ldots, e_{2}, e_{1}, e^{\prime}, e, \ldots
$$

or

$$
e, e^{\prime}, e_{1}, e_{2}, \ldots, e_{m-1}, e_{m}, e_{m-1}, \ldots, e_{2}, e_{1}, e^{\prime}, e, \ldots
$$

each of these cases is impossible.

We say that $\Gamma$ is $z$-knotted if it contains only one pair of zigzags $Z, Z^{-1}$; in other words, there is a single zigzag up to reversing. 


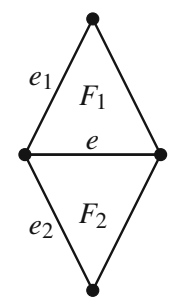

Fig. 4 Zigzags passing through the common edge of two faces

Lemma 2.1 If $\Gamma$ is z-knotted, then each of the two zigzags passes through every edge twice. Conversely, if $\Gamma$ contains a zigzag passing through every edge twice, then it is z-knotted.

This statement can be found in $[3,4]$, but some arguments from its proof will be used in what follows. For this reason, we present this proof below.

Proof of Lemma 2.1 Every edge $e$ is contained in precisely two distinct faces $F_{1}$ and $F_{2}$. We fix a vertex on $e$ and denote by $e_{i}, i=1,2$, the edge in $F_{i}$ containing this fixed vertex and distinct from $e$ (Fig. 4). Let $Z_{i}(e), i=1,2$, be the zigzag containing the sequence $e, e_{i}$.

If $\Gamma$ is $z$-knotted, i.e., it contains only one pair of zigzags $Z, Z^{-1}$, then each of these zigzags coincides with every $Z_{i}(e)$ or its reverse. This implies that it passes through $e$ twice.

If a zigzag passes through each edge twice, then it coincides with every $Z_{i}(e)$ or its reverse for all edges $e$. This is possible only in the case when $\Gamma$ is $z$-knotted.

\section{Main Result}

Let $\Gamma$ and $\Gamma^{\prime}$ be triangulations of (connected closed 2-dimensional) surfaces $M$ and $M^{\prime}$, respectively. Suppose that $F$ is a face in $\Gamma$ and $F^{\prime}$ is a face in $\Gamma^{\prime}$. By our assumption, $F$ and $F^{\prime}$ both are homeomorphic to a closed 2-dimensional disc and each of the boundaries $\partial F$ and $\partial F^{\prime}$ is the sum of three edges. Let $g: \partial F \rightarrow \partial F^{\prime}$ be a homeomorphism transferring every vertex of $F$ to a vertex of $F^{\prime}$, i.e., if $v_{i}, i \in\{1,2,3\}$, are the vertices of $F$, then $v_{i}^{\prime}=g\left(v_{i}\right), i \in\{1,2,3\}$, are the vertices of $F^{\prime}$. In what follows, such boundary homeomorphisms will be called special.

We define the connected sum $\Gamma \#_{g} \Gamma^{\prime}$. We remove the interiors of $F$ and $F^{\prime}$ from $M$ and $M^{\prime}$ (respectively) and glue together the boundaries $\partial F$ and $\partial F^{\prime}$ so that every $v_{i}$ is identified with $v_{i}^{\prime}$. We get a triangulation of the connected sum $M \# M^{\prime}$ which will be denoted by $\Gamma \#_{g} \Gamma^{\prime}$. The vertex set of $\Gamma \#_{g} \Gamma^{\prime}$ is the union of the vertex sets of $\Gamma$ and $\Gamma^{\prime}$, where every $v_{i}$ is identified with $v_{i}^{\prime}$, and the edge set is the union of the edge sets of $\Gamma$ and $\Gamma^{\prime}$, where the edge containing $v_{i}, v_{j}$ is identified with the edge containing $v_{i}^{\prime}, v_{j}^{\prime}$. Every face of $\Gamma \#_{g} \Gamma^{\prime}$ is a face of $\Gamma$ or $\Gamma^{\prime}$ different from $F, F^{\prime}$.

Note that for other special homeomorphism $h: \partial F \rightarrow \partial F^{\prime}$ the graph $\Gamma \#_{h} \Gamma^{\prime}$ is not necessarily isomorphic to $\Gamma \#_{g} \Gamma^{\prime}$. See Figs. 9 and 10 for non-isomorphic connected sums of 3-gonal bipyramids. 
Using the connected sums of triangulations, we describe the concept of shredding of a triangulation. As above, we suppose that $\Gamma$ is a triangulation of a surface $M$. Let $F_{1}, \ldots, F_{k}$ be mutually distinct faces of $\Gamma$ and let $\Gamma_{1}, \ldots, \Gamma_{k}$ be triangulations of the sphere $\mathbb{S}^{2}$. For each $i \in\{1, \ldots, k\}$ we take a face $F_{i}^{\prime}$ in $\Gamma_{i}$ and a special homeomorphism $g_{i}: \partial F_{i} \rightarrow \partial F_{i}^{\prime}$. The connected sum

$$
\left(\left(\left(\Gamma \#_{g_{1}} \Gamma_{1}\right) \#_{g_{2}} \Gamma_{2}\right) \cdots\right) \#_{g_{k}} \Gamma_{k}
$$

is a triangulation of $M$, where every $F_{i}$ is replaced by a triangulation of a 2-dimensional disc. Every triangulation of $M$ obtained from $\Gamma$ in such a way is said to be a shredding of $\Gamma$.

Theorem 3.1 Every triangulation $\Gamma$ of any connected closed 2-dimensional surface admits a $z$-knotted shredding. Suppose that $\Gamma$ contains precisely $2 m$ zigzags, i.e., $m$ zigzags up to reversing, and $m>1$. Then there are $z$-knotted triangulations $\Gamma_{1}, \ldots, \Gamma_{k}$ of the sphere $\mathbb{S}^{2}$ such that $k \leq m-1$ and the connected sum (1) is z-knotted.

\section{4 z-Monodromy}

Let $\Gamma$ be a triangulation and let $F$ be a face in $\Gamma$ whose vertices are denoted by $a, b, c$. Let also $\Omega(F)$ be the set consisting of all oriented edges of $F$. Then

$$
\Omega(F)=\{a b, b c, c a, a c, c b, b a\},
$$

where $x y$ is the edge from $x \in\{a, b, c\}$ to $y \in\{a, b, c\}$. If $e$ is the edge $x y$, then we write $-e$ for the edge $y x$.

Consider the permutation

$$
D_{F}=(a b, b c, c a)(a c, c b, b a)
$$

on the set $\Omega(F)$ (which is the composition of two commuting 3-cycles). If $x, y, z$ are three mutually distinct vertices of $F$, then $D_{F}(x y)=y z$. The equality $D_{F}(e)=e^{\prime}$ implies that $D_{F}\left(-e^{\prime}\right)=-e$.

For any $e \in \Omega(F)$ we take $e_{0} \in \Omega(F)$ such that $D_{F}\left(e_{0}\right)=e$ and consider the zigzag $Z$ containing the sequence $e_{0}, e$ (recall that every zigzag is completely determined by any pair of consecutive edges). If $e^{\prime}$ is the first element of $\Omega(F)$ contained in the zigzag $Z$ after $e$, then we define $M_{F}(e)=e^{\prime}$, Fig. 5. The transformation $M_{F}$ of $\Omega(F)$ will be called the z-monodromy associated to the face $F$.

Example 4.1 Let $e_{1}, \ldots, e_{n}$ be a zigzag of $\Gamma$ (we consider this zigzag as a cyclic sequence of non-oriented edges). For every $i \leq n-1$ we denote by $F_{i}$ the face containing the edges $e_{i}$ and $e_{i+1}$ and we write $F_{n}$ for the face which contains $e_{n}$ and $e_{1}$. It is easy to see that this zigzag is edge-simple if and only if the faces $F_{1}, \ldots, F_{n}$ are mutually distinct. In this case, we have $M_{F_{i}}\left(e_{i+1}\right)=e_{i}$ for every $i \leq n-1$ and $M_{F_{n}}\left(e_{1}\right)=e_{n}$, where $e_{1}, \ldots, e_{n}$ are considered as the edges oriented according to the direction of the zigzag. Therefore, if all zigzags of $\Gamma$ are edge-simple, then 


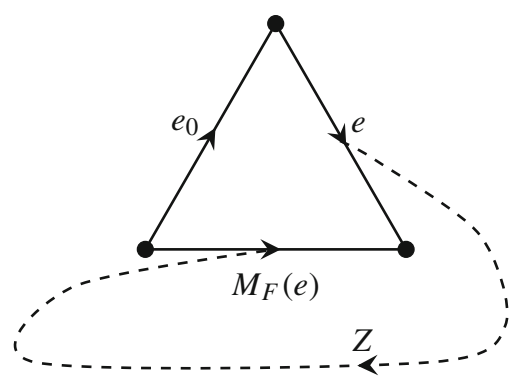

Fig. 5 -monodromy

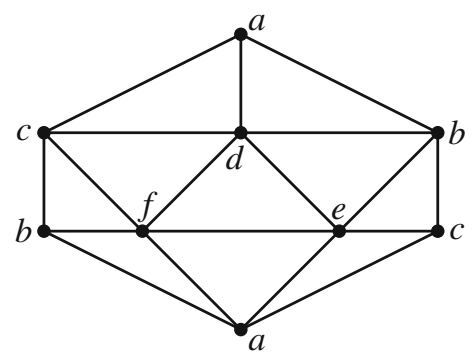

Fig. 6 Triangulation of the real projective plane with simple zigzags

$M_{F}=\left(D_{F}\right)^{-1}$ for every face $F$. Conversely, if the latter equality holds for every face $F$, then for any zigzag $e_{1}, \ldots, e_{n}$ the corresponding faces $F_{1}, \ldots, F_{n}$ are mutually distinct which implies that all zigzags are edge-simple. So, we have $M_{F}=\left(D_{F}\right)^{-1}$ for every face $F$ if and only if each zigzag of $\Gamma$ is edge-simple. All zigzags are simple (and consequently edge-simple) in the following triangulations:

- tetrahedrons, octahedrons and icosahedrons (three Platonic solids whose faces are triangles),

- the torus triangulation obtained from a square grid with diagonals in the same direction,

- the triangulation of the real projective plane presented in Fig. 6.

In Example 5.3, we show that all zigzags in a ( $2 k)$-gonal bipyramid are edge-simple if $k$ is even. Note that these zigzags are simple only for $k=2$ (the octahedron case).

Denote by $\mathcal{Z}(F)$ the set of all zigzags containing the sequences $e, D_{F}(e)$ with $e \in \Omega(F)$.

Lemma 4.2 The following assertions are fulfilled:

(1) A zigzag belongs to $\mathcal{Z}(F)$ if and only if it contains at least one edge of $F$.

(2) If a zigzag belongs to $\mathcal{Z}(F)$, then the same holds for the reversed zigzag.

(3) $|\mathcal{Z}(F)|$ is equal to 2 or 4 or 6 .

Proof (1) Suppose that a zigzag passes through the edge of $F$ containing vertices $x, y \in\{a, b, c\}$ and goes from $x$ to $y$. Then one of the following possibilities is 
realized: this zigzag contains the sequence $x y, D_{F}(x y)$, or it contains a sequence $e, x y$ such that $D_{F}(e)=x y$. In each of these cases, the zigzag belongs to $\mathcal{Z}(F)$.

(2) If a zigzag belongs to $\mathcal{Z}(F)$, then it contains a sequence $e, e^{\prime}$, where $e \in$ $\Omega(F)$ and $e^{\prime}=D_{F}(e)$. The reversed zigzag contains the sequence $-e^{\prime},-e$. Since $D_{F}\left(-e^{\prime}\right)=-e$, it belongs to $\mathcal{Z}(F)$.

(3) The set $\Omega(F)$ consists of 6 elements, but for some distinct $e, e^{\prime} \in \Omega(F)$ the zigzags containing the sequences $e, D_{F}(e)$ and $e^{\prime}, D_{F}\left(e^{\prime}\right)$ can be coincident. In this case, the reversed zigzags also are coincident.

We say that $\Gamma$ is locally $z$-knotted for $F$ if $|\mathcal{Z}(F)|=2$. By Lemma 4.2, this holds if and only if there is a single pair of zigzags $Z, Z^{-1}$ containing edges of $F$.

Lemma 4.3 If $\Gamma$ is locally z-knotted for $F$, then every zigzag from $\mathcal{Z}(F)$ passes through each edge of $F$ twice. Conversely, if there is a zigzag passing through each edge of $F$ twice, then $\Gamma$ is locally $z$-knotted for $F$.

Proof Similar to the proof of Lemma 2.1.

The main result of this section is a description of all possibilities for the $z$ monodromy.

Theorem 4.4 For the z-monodromy $M_{F}$ one of the following possibilities is realized:

(M1) $M_{F}$ is the identity,

(M2) $M_{F}=D_{F}$,

(M3) $M_{F}=\left(-e_{1}, e_{2}, e_{3}\right)\left(-e_{3},-e_{2}, e_{1}\right)$, where $\left(e_{1}, e_{2}, e_{3}\right)$ is one of the cycles in the permutation $D_{F}$,

(M4) $M_{F}=\left(e_{1},-e_{2}\right)\left(e_{2},-e_{1}\right)$, where $\left(e_{1}, e_{2}, e_{3}\right)$ is one of the cycles in $D_{F}\left(e_{3}\right.$ and $-e_{3}$ are fixed points),

(M5) $M_{F}=\left(D_{F}\right)^{-1}$,

(M6) $M_{F}=\left(-e_{1}, e_{2}, e_{3}\right)\left(-e_{3},-e_{2}, e_{1}\right)$, where $\left(e_{1}, e_{2}, e_{3}\right)$ is one of the cycles in the permutation $\left(D_{F}\right)^{-1}$,

(M7) $M_{F}=\left(e_{1}, e_{2}\right)\left(-e_{1},-e_{2}\right)$, where $\left(e_{1}, e_{2}, e_{3}\right)$ is one of the cycles in $D_{F}\left(e_{3}\right.$ and $-e_{3}$ are fixed points).

The triangulation $\Gamma$ is locally z-knotted for $F$ if and only if one of the cases (M1)-(M4) is realized.

In the next section, we give an example for each of the seven possibilities described in Theorem 4.4.

Lemma 4.5 The following assertions are fulfilled:

(1) The equality $M_{F}(e)=e^{\prime}$ implies that $M_{F}\left(-e^{\prime}\right)=-e$.

(2) $M_{F}$ is bijective.

(3) $M_{F}(e) \neq-e$ for every $e \in \Omega(F)$.

(4) The length of every cycle in the permutation $M_{F}$ is not greater than 3. 
Proof (1) Let $e \in \Omega(F)$. Consider $e_{0} \in \Omega(F)$ satisfying $D_{F}\left(e_{0}\right)=e$. If $Z$ is the zigzag containing the sequence $e_{0}, e$, then

$$
e^{\prime}=M_{F}(e) \text { and } e_{0}^{\prime}=D_{F} M_{F}(e)
$$

are the next two elements of $\Omega(F)$ in this zigzag. Observe that $D_{F}\left(-e_{0}^{\prime}\right)=-e^{\prime}$. The reversed zigzag $Z^{-1}$ contains the sequence $-e_{0}^{\prime},-e^{\prime}$ and $-e$ is the first element of $\Omega(F)$ contained in $Z^{-1}$ after $-e^{\prime}$. This means that $M_{F}\left(-e^{\prime}\right)=-e$.

(2) It is sufficient to show that $M_{F}$ is injective. Suppose that $M_{F}(e)=M_{F}\left(e^{\prime}\right)=e^{\prime \prime}$. By (1), we have $-e=M_{F}\left(-e^{\prime \prime}\right)=-e^{\prime}$ which implies that $e=e^{\prime}$.

(3) Let $e$ and $e_{0}$ be as in the proof of (1). If $M_{F}(e)=-e$, then the zigzag containing the sequence $e_{0}, e$ contains also the sequence $-e, D_{F}(-e)$. Since $D_{F}(-e)=-e_{0}$, this zigzag contains $e_{0}, e$ together with the reversed sequences $-e,-e_{0}$ which is impossible.

(4) Suppose that the permutation $M_{F}$ contains a cycle of the length greater than 3. Let $e_{1}, e_{2}, e_{3} \in \Omega(F)$ be consecutive elements in this cycle. Then

$$
M_{F}\left(e_{1}\right)=e_{2}, \quad M_{F}\left(e_{2}\right)=e_{3}, \quad M_{F}\left(e_{3}\right) \neq e_{1}
$$

and we have $M_{F}\left(e_{3}\right) \neq-e_{3}$ by (3). Therefore, $M_{F}\left(e_{3}\right)$ is equal to $-e_{1}$ or $-e_{2}$. By (1), the equality $M_{F}\left(e_{3}\right)=-e_{2}$ implies that $M_{F}\left(e_{2}\right)=-e_{3}$ which is impossible. So, we have $M_{F}\left(e_{3}\right)=-e_{1}$. Then $M_{F}\left(e_{1}\right)=-e_{3}$. The latter means that $e_{2}=-e_{3}$ which contradicts $M_{F}\left(e_{2}\right)=e_{3}$ by (3).

Using Lemma 4.5, we show that $M_{F}$ is one of the permutations (M1)-(M7). Suppose that $M_{F}$ is not the identity.

Consider the case when $M_{F}$ contains a 3-cycle $C$. By (3), this cycle does not contain pairs of type $e,-e$. This implies the existence of $e_{1}, e_{2}, e_{3} \in \Omega(F)$ such that $\left(e_{1}, e_{2}, e_{3}\right)$ is a cycle in $D_{F}$ or $\left(D_{F}\right)^{-1}$ and

$$
C=\left(e_{1}, e_{2}, e_{3}\right) \text { or } C=\left(-e_{1}, e_{2}, e_{3}\right) \text {. }
$$

Then (1) shows that

$$
M_{F}=\left(e_{1}, e_{2}, e_{3}\right)\left(-e_{3},-e_{2},-e_{1}\right) \text { or } M_{F}=\left(-e_{1}, e_{2}, e_{3}\right)\left(-e_{3},-e_{2}, e_{1}\right)
$$

We get the permutation (M2) or (M5) in the first case and (M3) or (M6) in the second.

If there is no 3-cycle in $M_{F}$, then it contains a transposition $T$. By (3), this transposition is not of type $(e,-e)$. Then there exist $e_{1}, e_{2}, e_{3} \in \Omega(F)$ such that $\left(e_{1}, e_{2}, e_{3}\right)$ is one of the cycles in $D_{F}$ and

$$
T=\left(e_{1}, e_{2}\right) \text { or } T=\left(e_{1},-e_{2}\right) .
$$

It follows from (1) that $M_{F}$ contains also the transposition $\left(-e_{1},-e_{2}\right)$ or the transposition $\left(e_{2},-e_{1}\right)$, respectively. Then (3) implies that $M_{F}$ leaves fixed $e_{3}$ and $-e_{3}$. So, we get (M4) or (M7). 
Lemma 4.6 The triangulation $\Gamma$ is locally z-knotted for $F$ if and only if $D_{F} M_{F}$ is the composition of two distinct commuting 3-cycles.

Proof Let $e, e_{0} \in \Omega(F)$ and $D_{F}\left(e_{0}\right)=e$. Consider the zigzag $Z$ containing the sequence $e_{0}, e$. If $e^{\prime}, e_{0}^{\prime} \in \Omega(F), D_{F}\left(e_{0}^{\prime}\right)=e^{\prime}$ and $Z$ contains the sequence $e_{0}^{\prime}, e^{\prime}$, then we write $\left[e^{\prime}, M_{F}\left(e^{\prime}\right)\right]$ for the part of $Z$ between $e^{\prime}$ and $M_{F}\left(e^{\prime}\right)$. The zigzag $Z$ is the cyclic sequence

$$
\left[e, M_{F}(e)\right],\left[D_{F} M_{F}(e), M_{F} D_{F} M_{F}(e)\right], \ldots,\left[\left(D_{F} M_{F}\right)^{m-1}(e), M_{F}\left(D_{F} M_{F}\right)^{m-1}(e)\right],
$$

where $m$ is the smallest non-zero number satisfying $\left(D_{F} M_{F}\right)^{m}(e)=e$. Since

$$
e, D_{F} M_{F}(e), \ldots,\left(D_{F} M_{F}\right)^{m-1}(e)
$$

are mutually distinct, the same holds for

$$
M_{F}(e), M_{F} D_{F} M_{F}(e), \ldots, M_{F}\left(D_{F} M_{F}\right)^{m-1}(e)
$$

Consider the sets

$$
\mathcal{X}=\left\{e, D_{F} M_{F}(e), \ldots,\left(D_{F} M_{F}\right)^{m-1}(e)\right\}
$$

and

$$
\mathcal{Y}=\left\{-M_{F}(e),-M_{F} D_{F} M_{F}(e), \ldots,-M_{F}\left(D_{F} M_{F}\right)^{m-1}(e)\right\}
$$

If $e^{\prime}$ belongs to $\mathcal{X} \cap \mathcal{Y}$ and $D_{F}\left(e^{\prime \prime}\right)=e^{\prime}$, then $D_{F}\left(-e^{\prime}\right)=-e^{\prime \prime}$ and $Z$ is a cyclic sequence of type

$$
\ldots,\left[*, e^{\prime \prime}\right],\left[e^{\prime}, *\right], \ldots,\left[*,-e^{\prime}\right],\left[-e^{\prime \prime}, *\right], \ldots ;
$$

in other words, the zigzag $Z$ is self-reversed which is impossible. So, $\mathcal{X} \cap \mathcal{Y}=\emptyset$. This implies that $m \leq 3$ (indeed, if $m>3$, then $\mathcal{X}$ and $\mathcal{Y}$ both contain more that three elements and have a non-empty intersection). Our zigzag

$$
Z=\left[e_{1}, M_{F}\left(e_{1}\right)\right], \ldots,\left[e_{m}, M_{F}\left(e_{m}\right)\right], \quad e_{i}=\left(D_{F} M_{F}\right)^{i-1}(e)
$$

corresponds to the $m$-cycle $C=\left(e_{1}, \ldots, e_{m}\right)$ in the permutation $D_{F} M_{F}$ and the reversed zigzag

$$
Z^{-1}=\left[-M_{F}\left(e_{m}\right),-e_{m}\right], \ldots,\left[-M_{F}\left(e_{1}\right),-e_{1}\right]
$$

corresponds to the $m$-cycle

$$
C^{\prime}=\left(-M_{F}\left(e_{m}\right), \ldots,-M_{F}\left(e_{1}\right)\right)
$$




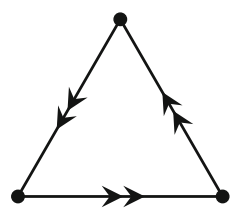

(a)

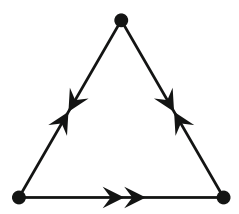

(b)

Fig. 7 Two types of faces in triangulations

(if $m=1$, then $e_{1}$ and $-M_{F}\left(e_{1}\right)$ both are fixed points of $D_{F} M_{F}$ ).

Suppose that $m=3$. Then $D_{F} M_{F}$ is the composition of the commuting 3-cycles $C$ and $C^{\prime}$. Also, we have $\Omega(F)=\mathcal{X} \cup \mathcal{Y}$ and every element of $\Omega(F)$ belongs to $\mathcal{X}$ or $\mathcal{Y}$. This means that $\mathcal{Z}(F)$ consists of $Z$ and $Z^{-1}$.

If $m<3$, then there are elements of $\Omega(F)$ which do not belong to $\mathcal{X} \cup \mathcal{Y}$. Such elements define zigzags distinct from $Z$ and $Z^{-1}$. Observe that $D_{F} M_{F}$ does not contain 3 -cycles in this case.

A direct verification shows that $D_{F} M_{F}$ is the composition of two distinct commuting 3-cycles if and only if $M_{F}$ is one of (M1)-(M4). Theorem 4.4 is proved.

Theorem 4.7 The triangulation $\Gamma$ is z-knotted if and only if for every face $F$ the $z$-monodromy $M_{F}$ is one of (M1)-(M4).

Proof If $\Gamma$ is $z$-knotted, then it is locally $z$-knotted for every face $F$ and each $z$ monodromy $M_{F}$ is one of (M1)-(M4) by Theorem 4.4.

Now, we suppose that for every face $F$ the $z$-monodromy $M_{F}$ is one of (M1)-(M4). It follows from Theorem 4.4 that $\Gamma$ is locally $z$-knotted for all faces. Let $F$ be a face of $\Gamma$ and let $\mathcal{Z}(F)=\left\{Z, Z^{-1}\right\}$. Then $Z$ passes through each edge of $F$. Therefore, if $F^{\prime}$ is a face adjacent to $F$ (i.e., intersecting $F$ in an edge), then $Z$ belongs to $\mathcal{Z}\left(F^{\prime}\right)$ by the statement (1) from Lemma 4.2. Since $\Gamma$ is locally $z$-knotted for $F^{\prime}$, we have $\mathcal{Z}\left(F^{\prime}\right)=\left\{Z, Z^{-1}\right\}$. The same holds for every face $F^{\prime}$ of $\Gamma$ by connectedness.

Remark 4.8 Suppose that the $z$-monodromy of a face $F$ is (M1) or (M2) and $Z \in \mathcal{Z}(F)$. Then $Z$ passes through each edge of $F$ twice in the same direction; in other words, it goes through three elements of $\Omega(F)$ twice. These elements form a cycle in $D_{F}$, see Fig. 7a.

In the case when the $z$-monodromy $M_{F}$ is (M3) or (M4), every zigzag from $\mathcal{Z}(F)$ goes through one edge twice in the same direction and through the remaining two edges twice in opposite directions, see Fig. 7b.

Remark 4.9 There is a one-to-one correspondence between cycles of the permutation $D_{F} M_{F}$ and zigzags belonging to $\mathcal{Z}(F)$ (see the proof of Lemma 4.6). An easy verification shows that $|\mathcal{Z}(F)|=6$ if $M_{F}$ is (M5) and we have $|\mathcal{Z}(F)|=4$ if $M_{F}$ is (M6) or (M7). 


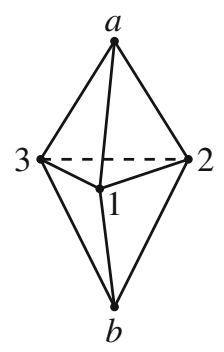

Fig. 8 3-gonal bipyramid

\section{Examples}

We describe zigzags in bipyramids and their connected sums (note that all zigzags will be presented as sequences of vertices) and give an example for each type of $z$ monodromy. In particular, we show that each of the $z$-monodromies (M1)-(M4) is realized in a $z$-knotted triangulation of the sphere $\mathbb{S}^{2}$.

\section{1 z-Monodromy in Bipyramids}

Consider the $n$-gonal bipyramid $B P_{n}, n \geq 3$, containing an $n$-gon whose vertices are denoted by $1, \ldots, n$ and connected with two disjoint vertices $a, b$ (see Fig. 8 for $n=3$ ). We describe the $z$-monodromy $M_{F}$ for the face $F$ containing the vertices $a, 1,2$. For other faces the $z$-monodromy is the same by the symmetry.

Example 5.1 (z-Monodromy of type (M3)) Suppose that $n=2 k+1$ and $k$ is odd. If $k=1$, then one of the zigzags is

$$
a, 1,2, b, 3,1, a, 2,3, b, 1,2, a, 3,1, b, 2,3 \text {. }
$$

For $k \geq 3$ this zigzag is

$$
\begin{aligned}
& a, 1,2, b, 3,4, \ldots, a, n-2, n-1, b, n, 1, a, 2,3, b, \ldots, a, n-1, n, \\
& \quad b, 1,2, a, 3,4, \ldots, b, n-2, n-1, a, n, 1, b, 2,3, a, \ldots, b, n-1, n .
\end{aligned}
$$

The zigzag passes through every edge twice and, by Lemma 2.1, the bipyramid is $z$-knotted. The face $F$ appears in the zigzag as follows

$$
a, 1,2, \ldots, 1, a, 2, \ldots, 1,2, a, \ldots
$$

which determines $M_{F}$ for three elements of $\Omega(F)$

$$
12 \rightarrow 1 a, \quad a 2 \rightarrow 12, \quad 2 a \rightarrow a 1
$$


By the statement (1) from Lemma 4.5, we have

$$
a 1 \rightarrow 21, \quad 21 \rightarrow 2 a, \quad 1 a \rightarrow a 2
$$

Let $e_{1}=12, e_{2}=2 a, e_{3}=a 1$. Then $\left(e_{1}, e_{2}, e_{3}\right)$ is one of the 3 -cycles in $D_{F}$. A simple verification shows that

$$
M_{F}=\left(-e_{1}, e_{2}, e_{3}\right)\left(-e_{3},-e_{2}, e_{1}\right)
$$

is of type (M3).

Example 5.2 ( $z$-Monodromy of type (M4)) Suppose that $n=2 k+1$ and $k$ is even. If $k=2$, then one of the zigzags is

$$
a, 1,2, b, 3,4, a, 5,1, b, 2,3, a, 4,5, b, 1,2, a, 3,4, b, 5,1, a, 2,3, b, 4,5 .
$$

For $k \geq 4$ we have

$$
\begin{aligned}
& a, 1,2, b, 3,4, a, \ldots, b, n-2, n-1, a, n, 1, b, 2,3, a, \ldots, a, n-1, n, \\
& \quad b, 1,2, a, 3,4, b, \ldots, a, n-2, n-1, b, n, 1, a, 2,3, b, \ldots, b, n-1, n .
\end{aligned}
$$

As in the previous example, this zigzag passes through every edge twice and the bipyramid is $z$-knotted. Let $e_{1}=2 a, e_{2}=a 1, e_{3}=12$. Then $\left(e_{1}, e_{2}, e_{3}\right)$ is one of the 3-cycles in $D_{F}$. The face $F$ appears in the zigzag as follows

$$
a, 1,2, \ldots, 1,2, a, \ldots, 1, a, 2, \ldots
$$

which implies that $M_{F}$ leaves fixed $e_{3}$ and transfers $e_{1}$ to $-e_{2}$ and $-e_{1}$ to $e_{2}$. Using the statement (1) from Lemma 4.5, we establish that

$$
M_{F}=\left(e_{1},-e_{2}\right)\left(e_{2},-e_{1}\right)
$$

is of type (M4).

Example 5.3 (z-Monodromy of type (M5)) Suppose that $n=2 k$ and $k$ is even. In the case when $k=2$, we get the octahedron whose zigzags are simple. Assume that $k \geq 4$. The set $\mathcal{Z}(F)$ contains precisely 8 zigzags:

- $a, 1,2, b, 3,4, \ldots, b, n-1, n$

- $b, 1,2, a, 3,4, \ldots, a, n-1, n$

- $1, a, 2,3, b, \ldots, a, n-2, n-1, b, n$

- $1, b, 2,3, a, \ldots, b, n-2, n-1, a, n$

and their reverses. These zigzags are not simple, but they are edge-simple. By Example 4.1, the $z$-monodromy of every face is of type (M5). 


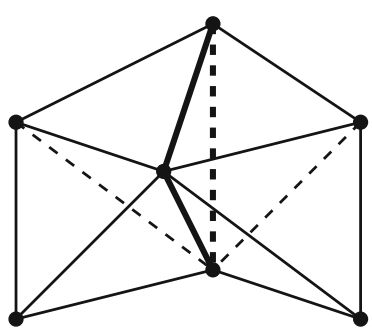

Fig. 9 Connected sum of two 3-gonal bipyramids with a face whose $z$-monodromy is of type (M2)

Example 5.4 (z-Monodromy of type (M7)). Suppose that $n=2 k$ and $k$ is an odd number greater than 1 . Then $\mathcal{Z}(F)$ is formed by the following two zigzags

$$
a, 1,2, b, 3,4, \ldots, a, n-1, n, b, 1,2, a, 3,4, \ldots, b, n-1, n
$$

and

$$
1, a, 2,3, \ldots, b, n-2, n-1, a, n, 1, b, 2,3, \ldots, a, n-2, n-1, b, n
$$

and their reverses. As in Example 5.2, we take $e_{1}=2 a, e_{2}=a 1, e_{3}=12$. Then $\left(e_{1}, e_{2}, e_{3}\right)$ is one of the 3 -cycles in $D_{F}$. The face $F$ appears in the zigzags as follows

$$
a, 1,2, \ldots, 1,2, a, \ldots \text { and } 1, a, 2, \ldots
$$

which implies that $M_{F}$ leaves fixed $e_{3}$ and transfers $e_{1}$ to $e_{2}$ and $-e_{1}$ to $-e_{2}$. By the statement (1) from Lemma 4.5,

$$
M_{F}=\left(e_{1}, e_{2}\right)\left(-e_{1},-e_{2}\right)
$$

is of type (M7).

\section{2 z-Monodromy in the Connected Sums of Bipyramids}

Let $B P_{n}$ be as in the previous subsection. Consider another bipyramid $B P_{n^{\prime}}$, where the vertices of the $n^{\prime}$-gon are denoted by $1^{\prime}, \ldots, n^{\prime}$ and we write $a^{\prime}, b^{\prime}$ for the remaining two vertices. Let $S$ and $S^{\prime}$ be the faces of the bipyramids containing the vertices $a, 1,2$ and $a^{\prime}, 1^{\prime}, 2^{\prime}$, respectively. We describe the $z$-monodromies for some faces in the connected sums $B P_{n} \#_{g} B P_{n^{\prime}}$, where $g: \partial S \rightarrow \partial S^{\prime}$ is a special homeomorphism. One of these sums for $n=n^{\prime}=3$ is presented in Fig. 9.

Example 5.5 (z-Monodromy of type (M2)). Suppose that $n=2 k+1$ and $n^{\prime}=2 k^{\prime}+1$, where $k$ and $k^{\prime}$ are odd. Let $\Gamma$ be the connected sum $B P_{n} \#_{g} B P_{n^{\prime}}$, where $g: \partial S \rightarrow \partial S^{\prime}$ satisfies

$$
g(a)=a^{\prime}, \quad g(1)=1^{\prime}, \quad g(2)=2^{\prime}
$$


(see Fig. 9 for $n=n^{\prime}=3$ ). The zigzag of $B P_{n}$ considered in Example 5.1 can be presented as the cyclic sequence $A, B, C$, where

$$
A=\{1,2, b, \ldots, 1, a\}, \quad B=\{a, 2, \ldots, b, 1,2\}, \quad C=\{2, a, \ldots, 1, b, 2, \ldots, a, 1\}
$$

are parts of the zigzag between two edges of the face $S$. Note that any two consecutive parts have the same vertex (for example, the parts $A$ and $B$ are joined in the vertex $a$ ). Similarly, one of the two zigzags of $B P_{n^{\prime}}$ is the cyclic sequence $A^{\prime}, B^{\prime}, C^{\prime}$, where

$$
A^{\prime}=\left\{1^{\prime}, 2^{\prime}, \ldots, 1^{\prime}, a^{\prime}\right\}, \quad B^{\prime}=\left\{a^{\prime}, 2^{\prime}, \ldots, 1^{\prime}, 2^{\prime}\right\}, \quad C^{\prime}=\left\{2^{\prime}, a^{\prime}, \ldots, a^{\prime}, 1^{\prime}\right\} .
$$

Consider the cyclic sequence

$$
A, C^{\prime-1}, B, A^{\prime}, C^{-1}, B^{\prime}
$$

where for any two consecutive parts $X, Y$ the last edge from $X$ is identified with the first edge from $Y$. A direct verification shows that this is a zigzag in $\Gamma$. This zigzag passes through each edge of $\Gamma$ twice (since it is obtained from a zigzag of $B P_{n}$ passing through all edges of $B P_{n}$ twice and a zigzag of $B P_{n^{\prime}}$ satisfying the same condition). Lemma 2.1 implies that $\Gamma$ is $z$-knotted. Let $F$ be the face of $\Gamma$ containing the vertices $b, 1,2$ and let $e_{1}=12, e_{2}=2 b, e_{3}=b 1$. Since this face appears in the zigzag as follows

$$
1,2, b, \ldots, b, 1,2, \ldots, 2, b, 1, \ldots
$$

the $z$-monodromy $M_{F}$ contains the 3-cycle $\left(e_{1}, e_{2}, e_{3}\right)$. By the statement (1) from Lemma 4.5,

$$
M_{F}=\left(e_{1}, e_{2}, e_{3}\right)\left(-e_{3},-e_{2},-e_{1}\right)=D_{F}
$$

is of type (M2).

Example 5.6 (z-Monodromy of type (M1)). Suppose that $n=2 k$ and $n^{\prime}=2 k^{\prime}$, where $k$ and $k^{\prime}$ are odd number greater than 1 . Denote by $\Gamma$ the connected sum $B P_{n} \#{ }_{g} B P_{n^{\prime}}$, where $g: \partial S \rightarrow \partial S^{\prime}$ satisfies

$$
g(a)=2^{\prime}, \quad g(1)=a^{\prime}, \quad g(2)=1^{\prime} .
$$

By Example 5.4, the set $\mathcal{Z}(S)$ is formed by the zigzags

$$
a, 1,2, b, 3,4, \ldots, a, n-1, n, b, 1,2, a, 3,4, \ldots, b, n-1, n
$$

and

$$
1, a, 2,3, \ldots, b, n-2, n-1, a, n, 1, b, 2,3, \ldots, a, n-2, n-1, b, n
$$


and their reverses. The first zigzag is the cyclic sequence $A, B$, where

$$
A=\{1,2, \ldots, 1,2\} \text { and } B=\{2, a, 3, \ldots, n, a, 1\}
$$

are parts of the zigzag between two edges of the face $S$ ( $A$ is joined with $B$ in the vertex 2 and $B$ is joined with $A$ in the vertex 1 ). The second zigzag passes once through the edges $a 1$ and $a 2$, and it does not contain the edge 12. We rewrite this zigzag as

$$
C=\{a, 2,3, \ldots, 2,3, a, \ldots, n, 1, a\}
$$

Similarly, $\mathcal{Z}\left(S^{\prime}\right)$ consists of four zigzags: one of them is $A^{\prime}, B^{\prime}$, the second is $C^{\prime}$, where

$$
A^{\prime}=\left\{1^{\prime}, 2^{\prime}, \ldots, 1^{\prime}, 2^{\prime}\right\}, \quad B^{\prime}=\left\{2^{\prime}, a^{\prime}, \ldots, a^{\prime}, 1^{\prime}\right\}, \quad C^{\prime}=\left\{a^{\prime}, 2^{\prime}, \ldots, 1^{\prime}, a^{\prime}\right\},
$$

and the remaining two zigzags are their reverses. Then

$$
A, C^{\prime-1}, C^{-1}, A^{\prime}, B, B^{\prime}
$$

(as in the previous example, for any two consecutive parts $X, Y$ the last edge from $X$ is identified with the first edge from $Y$ ) is a zigzag in $\Gamma$. This zigzag passes through all edges of $\Gamma$ twice (indeed, the sequence $A, B, C$ contains every edge of $B P_{n}$ twice and $A^{\prime}, B^{\prime}, C^{\prime}$ contains every edge of $B P_{n^{\prime}}$ twice). Therefore, $\Gamma$ is $z$-knotted. Let $F$ be the face of $\Gamma$ containing the vertices $a, 2,3$. This face appears in the zigzag as follows

$$
\ldots, a, 3,2, \ldots, 3,2, a, \ldots, 2, a, 3, \ldots
$$

which implies that $M_{F}$ is the identity.

Example 5.7 (z-Monodromy of type (M6)) As in Example 5.5, we suppose that $n=$ $2 k+1$ and $n^{\prime}=2 k^{\prime}+1$, where $k$ and $k^{\prime}$ are odd. Consider the connected sum $B P_{n} \#_{g} B P_{n^{\prime}}$, where $g: \partial S \rightarrow \partial S^{\prime}$ satisfies

$$
g(a)=1^{\prime}, \quad g(1)=2^{\prime}, \quad g(2)=a^{\prime}
$$

(see Fig. 10 for $n=n^{\prime}=3$ ). Note that this graph is not isomorphic to the graph from Example 5.5.

Recall that the single zigzags (up to reversing) in $B P_{n}$ and $B P_{n^{\prime}}$ are the cyclic sequences $A, B, C$ and $A^{\prime}, B^{\prime}, C^{\prime}$ (respectively), where

$$
\begin{aligned}
A & =\{1,2, b, \ldots, 1, a\}, \quad B=\{a, 2, \ldots, b, 1,2\}, \quad C=\{2, a, \ldots, 1, b, 2, \ldots, a, 1\}, \\
A^{\prime} & =\left\{1^{\prime}, 2^{\prime}, \ldots, 1^{\prime}, a^{\prime}\right\}, \quad B^{\prime}=\left\{a^{\prime}, 2^{\prime}, \ldots, 1^{\prime}, 2^{\prime},\right\}, \quad C^{\prime}=\left\{2^{\prime}, a^{\prime}, \ldots, a^{\prime}, 1^{\prime}\right\} .
\end{aligned}
$$

The cyclic sequences

$$
A, B^{\prime-1} \text { and } B, C^{\prime}, C, A^{\prime}
$$




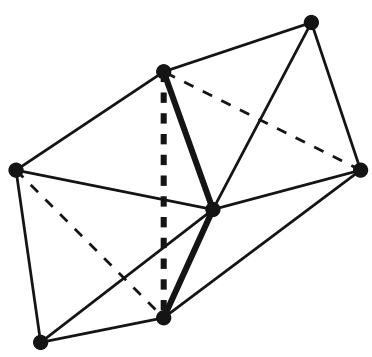

Fig. 10 Connected sum of two 3-gonal bipyramids with a face whose z-monodromy is of type (M6)

define zigzags in $B P_{n} \#_{g} B P_{n^{\prime}}$ (the corresponding edges in consecutive parts are identified). Let $F$ be the face of the connected sum which contains the vertices $b, 1,2$ and let $e_{1}=b 1, e_{2}=2 b, e_{3}=12$. Then $\left(e_{1}, e_{2}, e_{3}\right)$ is one of the 3 -cycles in $\left(D_{F}\right)^{-1}$. The face $F$ appears in the zigzags as follows

$$
1,2, b, \ldots \text { and } \ldots, b, 1,2, \ldots, 1, b, 2, \ldots
$$

which determines $M_{F}$ on three elements of $\Omega(F)$

$$
e_{2} \rightarrow e_{3}, \quad e_{3} \rightarrow-e_{1}, \quad-e_{2} \rightarrow e_{1}
$$

The statement (1) from Lemma 4.5 implies that

$$
-e_{3} \rightarrow-e_{2}, \quad e_{1} \rightarrow-e_{3}, \quad-e_{1} \rightarrow e_{2}
$$

Therefore,

$$
M_{F}=\left(-e_{1}, e_{2}, e_{3}\right)\left(-e_{3},-e_{2}, e_{1}\right)
$$

is of type (M6).

\section{Gluing Lemma}

Let $\Gamma$ and $\Gamma^{\prime}$ be triangulations. Let also $F$ and $F^{\prime}$ be faces in $\Gamma$ and $\Gamma^{\prime}$, respectively. Every special homeomorphism $g: \partial F \rightarrow \partial F^{\prime}$ induces a bijection between $\Omega(F)$ and $\Omega\left(F^{\prime}\right)$ which also will be denoted by $g$ and which sends every oriented edge $x y$ to the oriented edge $g(x) g(y)$. The following properties of the bijection $g: \Omega(F) \rightarrow \Omega\left(F^{\prime}\right)$ are obvious:

- $g(-e)=-g(e)$ for every $e \in \Omega(F)$,

- $g D_{F} g^{-1}=D_{F^{\prime}}$.

A face $S$ in a triangulation is said to be essential if every zigzag of this triangulation contains an edge from this face, or equivalently, every zigzag belongs to $\mathcal{Z}(S)$. It is clear that all faces in $z$-knotted triangulations are essential. Also, every face in a tetrahedron is essential. 
Lemma 6.1 The following assertions are fulfilled:

(1) Suppose that $F$ and $F^{\prime}$ are essential faces. Then the connected sum $\Gamma \#_{g} \Gamma^{\prime}$ is $z$ knotted if and only if $g M_{F} g^{-1} M_{F^{\prime}}$ is the composition of two distinct commuting 3-cycles.

(2) Suppose that $\Gamma^{\prime}$ is z-knotted and $g M_{F} g^{-1} M_{F^{\prime}}$ is the composition of two distinct commuting 3-cycles. Then $\Gamma \#_{g} \Gamma^{\prime}$ contains a zigzag $Z$ such that $\mathcal{Z}(S)=\left\{Z, Z^{-1}\right\}$ for every face $S$ in $\Gamma_{g} \Gamma^{\prime}$ corresponding to a face of $\Gamma^{\prime}$ distinct from $F^{\prime}$.

Proof For every $e \in \Omega(F)$ we consider the zigzag of $\Gamma$ containing the sequence $\left(D_{F}\right)^{-1}(e), e$ and $M_{F}(e)$ (see the definition of $z$-monodromy). As in the proof of Lemma 4.6, we write $\left[e, M_{F}(e)\right]$ for the part of this zigzag between $e$ and $M_{F}(e)$. Let $\mathcal{X}$ be the set of all $\left[e, M_{F}(e)\right]$, where $e \in \Omega(F)$. This set consists of 6 elements, and for every $X \in \mathcal{X}$ the reversed path $X^{-1}$ also belongs to $\mathcal{X}$ (indeed, for $X=\left[e, M_{F}(e)\right]$ we have $X^{-1}=\left[e^{\prime}, M_{F}\left(e^{\prime}\right)\right]$, where $\left.e^{\prime}=-M_{F}(e)\right)$.

Similarly, we denote by $\mathcal{X}^{\prime}$ the set of all $\left[e, M_{F^{\prime}}(e)\right]$, where $e \in \Omega\left(F^{\prime}\right)$ (note that the elements of $\mathcal{X}^{\prime}$ are contained in zigzags of $\left.\Gamma^{\prime}\right)$.

Let $e \in \Omega\left(F^{\prime}\right)$. Consider the cyclic sequence

$$
\begin{gathered}
{\left[e, M_{F^{\prime}}(e)\right],\left[g^{-1} M_{F^{\prime}}(e), M_{F} g^{-1} M_{F^{\prime}}(e)\right],} \\
{\left[g M_{F} g^{-1} M_{F^{\prime}}(e), M_{F^{\prime}}\left(g M_{F} g^{-1} M_{F^{\prime}}(e)\right],\right.} \\
\vdots \\
{\left[g^{-1} M_{F^{\prime}}\left(g M_{F} g^{-1} M_{F^{\prime}}\right)^{m-1}(e), M_{F} g^{-1} M_{F^{\prime}}\left(g M_{F} g^{-1} M_{F^{\prime}}\right)^{m-1}(e)\right],}
\end{gathered}
$$

where $m$ is the smallest non-zero number satisfying

$$
\left(g M_{F} g^{-1} M_{F^{\prime}}\right)^{m}(e)=e .
$$

In fact, this is a cyclic sequence

$$
X_{1}^{\prime}, X_{1}, \ldots, X_{m}^{\prime}, X_{m}
$$

where all $X_{i}$ belong to $\mathcal{X}$ and every $X_{i}^{\prime}$ is an element of $\mathcal{X}^{\prime}$. As in the examples from the previous section, for any two consecutive parts $A, B \in \mathcal{X} \cup \mathcal{X}^{\prime}$ in this sequence we identify the last edge from $A$ with the first edge from $B$ and get a zigzag in the connected sum $\Gamma \#_{g} \Gamma^{\prime}$. We denote this zigzag by $Z(e)$. We have

$$
X_{i} \neq X_{j}^{-1} \text { and } X_{i}^{\prime} \neq X_{j}^{\prime-1}
$$

for any pair $i, j \in\{1, \ldots, m\}$ (otherwise, the zigzag $Z(e)$ is self-reversed which is impossible). This implies that $m \leq 3$.

The zigzag $Z(e)$ corresponds to an $m$-cycle in the permutation $g M_{F} g^{-1} M_{F^{\prime}}$ and the reversed zigzag $Z(e)^{-1}$ is the cyclic sequence

$$
X_{m}^{-1}, X_{m}^{\prime-1}, \ldots, X_{1}^{-1}, X_{1}^{\prime-1}
$$


related to a different $m$-cycle in this permutation. Note that $Z(e)^{-1}$ coincides with $Z\left(e^{\prime}\right)$ for a certain $e^{\prime} \in \Omega\left(F^{\prime}\right)$. As in the proof of Lemma 4.6, we establish that the following two conditions are equivalent:

(A) the permutation $g M_{F} g^{-1} M_{F^{\prime}}$ is the composition of two distinct commuting 3cycles,

(B) for any $e, e^{\prime} \in \Omega\left(F^{\prime}\right)$ the zigzag $Z\left(e^{\prime}\right)$ coincides with $Z(e)$ or $Z(e)^{-1}$.

Now, we prove the statements (1) and (2).

(1) If the faces $F$ and $F^{\prime}$ both are essential, then every zigzag of $\Gamma$ or $\Gamma^{\prime}$ contains an edge from $F$ or $F^{\prime}$ (respectively). This implies that every zigzag in the connected sum $\Gamma \#_{g} \Gamma^{\prime}$ is of type $Z(e)$, where $e \in \Omega\left(F^{\prime}\right)$. Then (B) will be equivalent to the $z$-knottedness of the connected sum.

(2) Suppose that $\Gamma^{\prime}$ is $z$-knotted and the condition (A) holds. Then (B) also holds true and we assert that the zigzag $Z=Z(e), e \in \Omega\left(F^{\prime}\right)$ is as required.

The triangulation $\Gamma^{\prime}$ contains a single pair of zigzags $Z^{\prime}, Z^{\prime-1}$. As in the proof of Lemma 4.6, we have

$$
Z^{\prime}=X_{1}^{\prime}, X_{2}^{\prime}, X_{3}^{\prime} \text { and } Z^{\prime-1}=X_{3}^{\prime-1}, X_{2}^{\prime-1}, X_{1}^{\prime-1}
$$

where $X_{1}^{\prime}, X_{2}^{\prime}, X_{3}^{\prime}, X_{1}^{\prime-1}, X_{2}^{\prime-1}, X_{3}^{\prime-1}$ are mutually distinct elements of $\mathcal{X}^{\prime}$, in other words, the set $\mathcal{X}^{\prime}$ is formed by these elements. The condition (B) guarantees that every $X^{\prime} \in \mathcal{X}^{\prime}$ is contained in $Z$ or $Z^{-1}$.

By Lemma 2.1, each of the zigzags $Z^{\prime}, Z^{\prime-1}$ passes through every edge of $\Gamma^{\prime}$ twice. This implies that each of the zigzags $Z, Z^{-1}$ also passes through every edge of $\Gamma^{\prime}$ twice. Using arguments from the proof of Lemma 2.1, we show that there is no other zigzag of $\Gamma \# \#_{g} \Gamma^{\prime}$ containing edges from $\Gamma^{\prime}$. Therefore, $\mathcal{Z}(S)=\left\{Z, Z^{-1}\right\}$ for every face $S \neq F^{\prime}$ of $\Gamma^{\prime}$ considered as a face of the connected sum $\Gamma \# \#_{g} \Gamma^{\prime}$.

\section{Proof of Theorem 3.1}

Let $\Gamma$ be a triangulation that is not locally $z$-knotted for a certain face $F$.

Lemma 7.1 There is a z-knotted triangulation $\Gamma^{\prime}$ of the sphere $\mathbb{S}^{2}$ and a face $F^{\prime}$ in this triangulation such that $g M_{F} g^{-1} M_{F^{\prime}}$ is the composition of two distinct commuting 3-cycles for a certain special homeomorphism $g: \partial F \rightarrow \partial F^{\prime}$.

Proof By Theorem 4.4, the $z$-monodromy $M_{F}$ is one of (M5)-(M7).

Suppose that $M_{F}$ is (M5) or (M6). Then it is the composition of two distinct commuting 3-cycles. Let us take a $z$-knotted triangulation $\Gamma^{\prime}$ of $\mathbb{S}^{2}$ containing a face $F^{\prime}$ such that $M_{F^{\prime}}$ is the identity, for example, the triangulation from Example 5.6. Then

$$
g M_{F} g^{-1} M_{F^{\prime}}=g M_{F} g^{-1}
$$

is the composition of two distinct commuting 3-cycles for every special homeomorphism $g: \partial F \rightarrow \partial F^{\prime}$. 
Consider the case when $M_{F}$ is (M7), i.e.,

$$
M_{F}=\left(e_{1}, e_{2}\right)\left(-e_{1},-e_{2}\right),
$$

where $\left(e_{1}, e_{2}, e_{3}\right)$ is one of the cycles in $D_{F}$ and $e_{3},-e_{3}$ are fixed points. Let $\Gamma^{\prime}$ be the bipyramid $B P_{2 k+1}$, where $k$ is odd. For every face $F^{\prime}$ of $\Gamma^{\prime}$ we have

$$
M_{F^{\prime}}=\left(-e_{1}^{\prime}, e_{2}^{\prime}, e_{3}^{\prime}\right)\left(-e_{3}^{\prime},-e_{2}^{\prime}, e_{1}^{\prime}\right),
$$

where $\left(e_{1}^{\prime}, e_{2}^{\prime}, e_{3}^{\prime}\right)$ is one of the cycles in the permutation $D_{F^{\prime}}$, see Example 5.1. There is a special homeomorphism $g: \partial F \rightarrow \partial F^{\prime}$ satisfying $g\left(e_{i}\right)=e_{i}^{\prime}$ for every $i$. A direct verification shows that $g M_{F} g^{-1} M_{F^{\prime}}$ is the composition of two distinct commuting 3-cycles.

Let $\Gamma^{\prime}, F^{\prime}$ and $g: \partial F \rightarrow \partial F^{\prime}$ be as in Lemma 7.1. By the statement (2) from Lemma 6.1, the connected sum $\Gamma \#_{g} \Gamma^{\prime}$ contains a zigzag $Z$ such that $\mathcal{Z}(S)=\left\{Z, Z^{-1}\right\}$ for every face $S$ in $\Gamma \#_{g} \Gamma^{\prime}$ corresponding to a face of $\Gamma^{\prime}$ distinct from $F^{\prime}$. Therefore, $\Gamma \#_{g} \Gamma^{\prime}$ is locally $z$-knotted for all faces of $\Gamma^{\prime}$ distinct from $F^{\prime}$.

Lemma 7.2 Suppose that $S$ is a face of $\Gamma$ distinct from $F$. If $\Gamma$ is locally $z$-knotted for $S$, then $\Gamma \#_{g} \Gamma^{\prime}$ also is locally z-knotted for $S$.

Proof Let $Z_{S},\left(Z_{S}\right)^{-1}$ be a unique pair of zigzags in $\Gamma$ containing edges of $S$. By Lemma 4.3, each of these zigzags passes through every edge of $S$ twice.

If $Z_{S}$ and $\left(Z_{S}\right)^{-1}$ do not contain any edge from $F$, then they are zigzags in $\Gamma \#_{g} \Gamma^{\prime}$, and the connected sum is locally $z$-knotted for $S$ by Lemma 4.3.

If $Z_{S}$ and $\left(Z_{S}\right)^{-1}$ contain some edges of $F$, then they belong to $\mathcal{Z}(F)$. In the proof of Lemma 6.1, we consider the set $\mathcal{X}$ formed by all parts of the zigzags from $\mathcal{Z}(F)$ between $e \in \Omega(F)$ and $M_{F}(e)$. Recall that every $X \in \mathcal{X}$ is contained in $Z$ or $Z^{-1}$. Since $Z_{S}$ and $\left(Z_{S}\right)^{-1}$ belong to $\mathcal{Z}(F)$, they are cyclic sequences formed by at most three elements of $\mathcal{X}$. Each of $Z_{S},\left(Z_{S}\right)^{-1}$ passes through every edge of $S$ twice and the same holds for $Z$ and $Z^{-1}$. Lemma 4.3 implies that $\Gamma \#_{g} \Gamma^{\prime}$ is locally $z$-knotted for $S$.

So, the connected sum $\Gamma \#_{g} \Gamma^{\prime}$ has the following properties:

(1) it is locally $z$-knotted for all faces of $\Gamma^{\prime}$ distinct from $F^{\prime}$,

(2) if $\Gamma$ is locally $z$-knotted for a face $S \neq F$, then $\Gamma \#_{g} \Gamma^{\prime}$ is locally $z$-knotted for $S$.

If $\Gamma \#_{g} \Gamma^{\prime}$ is not locally $z$-knotted for a face $T$, then $T$ is a face of $\Gamma$ by (1). We apply the above arguments to the face $T$ in $\Gamma \#_{g} \Gamma^{\prime}$. Using (2), we construct recursively a shredding of $\Gamma$ which is locally $z$-knotted for every face. By Theorem 4.7, this shredding is $z$-knotted.

Suppose that $\Gamma$ contains precisely $2 m$ zigzags, i.e., $m$ zigzags up to reversing. If $\Gamma$ is not locally $z$-knotted for a face $F$, then $\mathcal{Z}(F)$ contains two or three zigzags up to reversing (Remark 4.9). Using the corresponding connected sum, we replace these zigzags by one zigzag and come to a triangulation with $m-1$ or $m-2$ zigzags up to reversing. So, we need at most $m-1$ times to get a $z$-knotted shredding of $\Gamma$. 


\section{Connected Sums of z-Knotted Triangulations}

By Theorem 4.4, there are precisely four types of faces in $z$-knotted triangulations. The corresponding $z$-monodromies are (M1)-(M4). In [8], these four types were described without $z$-monodromy. The main result of [8] determines all cases when the connected sum of two $z$-knotted triangulations is $z$-knotted. The proof given in [8] was a long case-by-case verification. Now, this result will be presented as a simple consequence of the statement (1) from Lemma 6.1.

Theorem 8.1 Let $\Gamma$ and $\Gamma^{\prime}$ be $z$-knotted triangulations. Then the following assertions are fulfilled:

(1) If $F$ is a face in $\Gamma$ such that $M_{F}=D_{F}$ (type (M2)), then for every face $F^{\prime}$ in $\Gamma^{\prime}$ and every special homeomorphism $g: \partial F \rightarrow \partial F^{\prime}$ the connected sum $\Gamma \#{ }_{g} \Gamma^{\prime}$ is z-knotted.

(2) Suppose that $F$ is a face in $\Gamma$ and $M_{F}$ is the identity (type (M1)). If $F^{\prime}$ is a face in $\Gamma^{\prime}$ such that the connected sum $\Gamma \#_{g} \Gamma^{\prime}$ is z-knotted for a certain special homeomorphism $g: \partial F \rightarrow \partial F^{\prime}$, then $M_{F^{\prime}}$ is $D_{F^{\prime}}$ or (M3). In these cases, the connected sum $\Gamma \#_{g} \Gamma^{\prime}$ is z-knotted for every special homeomorphism $g: \partial F \rightarrow$ $\partial F^{\prime}$.

(3) If $F$ is a face in $\Gamma, F^{\prime}$ is a face in $\Gamma^{\prime}$ and $M_{F}, M_{F^{\prime}}$ are (M3) or (M4), then there is a special homeomorphism $g: \partial F \rightarrow \partial F^{\prime}$ such that the connected sum $\Gamma \#_{g} \Gamma^{\prime}$ is z-knotted.

Proof Recall that every special homeomorphism $g: \partial F \rightarrow \partial F^{\prime}$ induces a bijection of $\Omega(F)$ to $\Omega\left(F^{\prime}\right)$. This bijection is also denoted by $g$.

(1) It is easy to see that $g D_{F} g^{-1}=D_{F^{\prime}}$. Therefore, $M_{F}=D_{F}$ implies that

$$
g M_{F} g^{-1} M_{F^{\prime}}=D_{F^{\prime}} M_{F^{\prime}}
$$

for every special homeomorphism $g: \partial F \rightarrow \partial F^{\prime}$. Since $\Gamma^{\prime}$ is $z$-knotted, $D_{F^{\prime}} M_{F^{\prime}}$ is the composition of two distinct commuting 3-cycles (Lemma 4.6). The statement (1) from Lemma 6.1 implies that the connected sum $\Gamma \#_{g} \Gamma^{\prime}$ is $z$-knotted.

(2) If $M_{F}$ is the identity, then

$$
g M_{F} g^{-1} M_{F^{\prime}}=M_{F^{\prime}}
$$

for every special homeomorphism $g: \partial F \rightarrow \partial F^{\prime}$. We get the composition of two distinct commuting 3-cycles only in the case when $M_{F^{\prime}}$ is $D_{F^{\prime}}$ or (M3).

(3) Suppose that $M_{F}$ and $M_{F^{\prime}}$ both are (M3), i.e.,

$$
M_{F}=\left(-e_{1}, e_{2}, e_{3}\right)\left(-e_{3},-e_{2}, e_{1}\right) \text { and } M_{F^{\prime}}=\left(-e_{1}^{\prime}, e_{2}^{\prime}, e_{3}^{\prime}\right)\left(-e_{3}^{\prime},-e_{2}^{\prime}, e_{1}^{\prime}\right) \text {, }
$$

where $\left(e_{1}, e_{2}, e_{3}\right)$ and $\left(e_{1}^{\prime}, e_{2}^{\prime}, e_{3}^{\prime}\right)$ are 3 -cycles in $D_{F}$ and $D_{F^{\prime}}$, respectively. There exists a special homeomorphism $g: \partial F \rightarrow \partial F^{\prime}$ which transfers $e_{1}, e_{2}, e_{3}$ to $e_{1}^{\prime}, e_{2}^{\prime}, e_{3}^{\prime}$ (respectively). Then $g M_{F} g^{-1}=M_{F^{\prime}}$ and 


$$
g M_{F} g^{-1} M_{F^{\prime}}=\left(M_{F^{\prime}}\right)^{2}=\left(M_{F^{\prime}}\right)^{-1}
$$

is the composition of two distinct commuting 3-cycles.

Consider the case when $F$ and $F^{\prime}$ both are (M4), i.e.,

$$
M_{F}=\left(e_{1},-e_{2}\right)\left(e_{2},-e_{1}\right) \text { and } M_{F^{\prime}}=\left(e_{1}^{\prime},-e_{2}^{\prime}\right)\left(e_{2}^{\prime},-e_{1}^{\prime}\right)
$$

where $\left(e_{1}, e_{2}, e_{3}\right)$ and $\left(e_{1}^{\prime}, e_{2}^{\prime}, e_{3}^{\prime}\right)$ are 3 -cycles in $D_{F}$ and $D_{F^{\prime}}$, respectively. We take a special homeomorphism $g: \partial F \rightarrow \partial F^{\prime}$ transferring $e_{1}, e_{2}, e_{3}$ to $e_{3}^{\prime}, e_{1}^{\prime}, e_{2}^{\prime}$ (respectively). Then

$$
g M_{F} g^{-1}=\left(e_{1}^{\prime},-e_{3}^{\prime}\right)\left(e_{3}^{\prime},-e_{1}^{\prime}\right)
$$

and

$$
g M_{F} g^{-1} M_{F^{\prime}}=\left(-e_{1}^{\prime}, e_{2}^{\prime}, e_{3}^{\prime}\right)\left(e_{1}^{\prime},-e_{2}^{\prime},-e_{3}^{\prime}\right)
$$

is the composition of two distinct commuting 3-cycles.

Suppose that $F$ is (M4) and $F^{\prime}$ is (M3), i.e.,

$$
M_{F}=\left(e_{1},-e_{2}\right)\left(e_{2},-e_{1}\right) \text { and } M_{F^{\prime}}=\left(-e_{1}^{\prime}, e_{2}^{\prime}, e_{3}^{\prime}\right)\left(-e_{3}^{\prime},-e_{2}^{\prime}, e_{1}^{\prime}\right),
$$

where $\left(e_{1}, e_{2}, e_{3}\right)$ and $\left(e_{1}^{\prime}, e_{2}^{\prime}, e_{3}^{\prime}\right)$ are 3 -cycles in $D_{F}$ and $D_{F^{\prime}}$, respectively. There is a special homeomorphism $g: \partial F \rightarrow \partial F^{\prime}$ sending $e_{1}, e_{2}, e_{3}$ to $e_{2}^{\prime}, e_{3}^{\prime}, e_{1}^{\prime}$ (respectively). Then

$$
g M_{F} g^{-1}=\left(e_{2}^{\prime},-e_{3}^{\prime}\right)\left(e_{3}^{\prime},-e_{2}^{\prime}\right)
$$

and

$$
g M_{F} g^{-1} M_{F^{\prime}}=\left(e_{1}^{\prime}, e_{2}^{\prime},-e_{2}^{\prime}\right)\left(-e_{1}^{\prime},-e_{3}^{\prime}, e_{3}^{\prime}\right)
$$

is the composition of two distinct commuting 3-cycles.

In each of these three cases, the connected sum $\Gamma \#_{g} \Gamma^{\prime}$ is $z$-knotted by the statement (1) from Lemma 6.1.

Open Access This article is licensed under a Creative Commons Attribution 4.0 International License, which permits use, sharing, adaptation, distribution and reproduction in any medium or format, as long as you give appropriate credit to the original author(s) and the source, provide a link to the Creative Commons licence, and indicate if changes were made. The images or other third party material in this article are included in the article's Creative Commons licence, unless indicated otherwise in a credit line to the material. If material is not included in the article's Creative Commons licence and your intended use is not permitted by statutory regulation or exceeds the permitted use, you will need to obtain permission directly from the copyright holder. To view a copy of this licence, visit http://creativecommons.org/licenses/by/4.0/. 


\section{References}

1. Coxeter, H.S.M.: Regular Polytopes, 3rd edn. Dover, New York (1973)

2. Crapo, H., Rosenstiehl, P.: On lacets and their manifolds. Discrete Math. 233(1-3), 299-320 (2001)

3. Deza, M.-M., Dutour Sikirić, M., Shtogrin, M.I.: Geometric Structure of Chemistry-Relevant Graphs: Zigzags and Central Circuit. Forum for Interdisciplinary Mathematics. Springer, New Delhi (2015)

4. Godsil, C., Royle, G.: Algebraic Graph Theory. Graduate Texts in Mathematics. Springer, New York (2001)

5. Lins, S.: Graph-encoded maps. J. Combin. Theory Ser. B 32(2), 171-181 (1982)

6. Lins, S., Oliveira-Lima, E., Silva, V.: A homological solution for the Gauss code problem in arbitrary surfaces. J. Combin. Theory Ser. B 98(3), 506-515 (2008)

7. Mohar, B., Thomassen, C.: Graphs on Surfaces. Johns Hopkins Studies in the Mathematical Sciences. Johns Hopkins University Press, Baltimore (2001)

8. Pankov, M., Tyc, A.: Connected sums of z-knotted triangulations. Eur. J. Comb. 80, 326-338 (2019)

9. Shank, H.: The theory of left-right paths. In: Penfold Street, A., Wallis, W.D. (eds.) Combinatorial Mathematics, vol. III. Lecture Notes in Mathematics, pp. 42-54. Springer, Berlin (1975)

Publisher's Note Springer Nature remains neutral with regard to jurisdictional claims in published maps and institutional affiliations. 